\title{
RHABDOMYOSARCOMA
}

natuire DISEASE

REVIEWS PRIMERS

Rhabdomyosarcoma (RMS) is the most common soft tissue sarcoma in children, in which tumour cells have a tendency for myogenic (that is, muscle cell like) differentiation. Two major subtypes exist: fusion-positive RMS (FPRMS) and fusion-negative RMS (FNRMS).

EPIDEMIOLOGY

RMS is considered a rare disease. In the United States, 350 individuals are diagnosed with RMS per year. This figure equates to an incidence of $\sim$. 5 patients per million individuals $<20$ years of age. Children with specific genetic disorders, including Li-Fraumeni, neurofibromatosis type 1 , DICER1 and Costello and Noonan syndromes, have an increased risk. Interestingly, children of Hispanic ethnicity have a decreased risk of RMS. Finally, environmental factors may increase the risk of RMS, including prenatal $\mathrm{X}$-ray exposures and parental recreational drug use.

\section{QUALITY OF LIFE}

Special consideration must be given to longterm quality of life in children with RMS, as the majority will be sucessfully treated. If possible, surgical interventions that negatively affect form or function should be avoided. The use of ionizing radiation should be carefully considered in patients who are classified as low risk, owing to the risk of radiotherapy toxicity and secondary malignancies. Despite these concerns,

knowledge gaps still exist in the long-term quality of life in survivors of RMS. Q 10,00

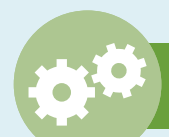

MECHANISMS

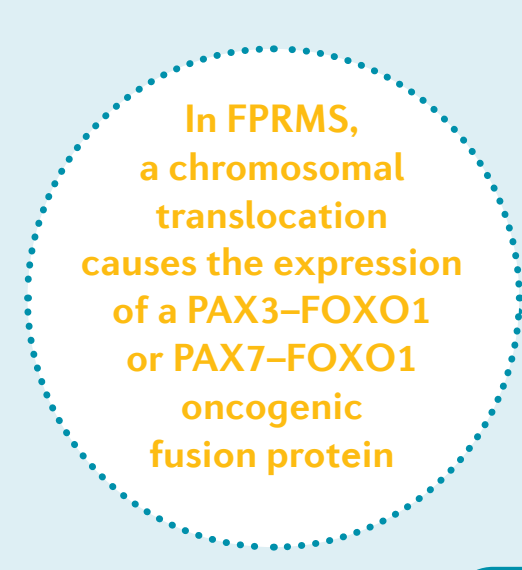

$\because$ $\because \ldots \ldots \ldots \ldots . . . .{ }^{\circ}$

PAX-FOXO1
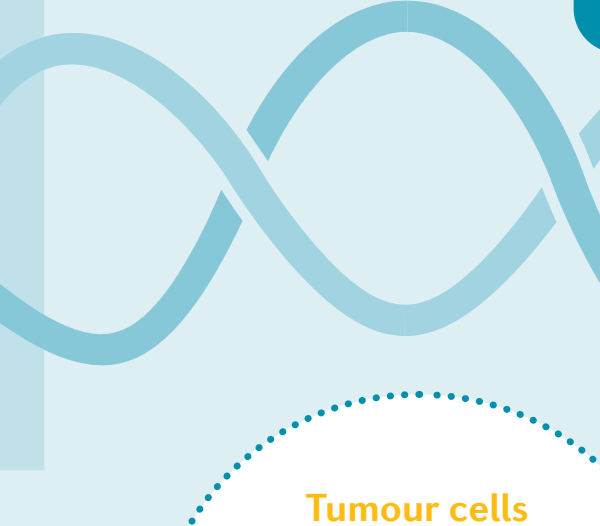

$\therefore . . \cdots$

Tumour cells

in FNRMS express

no fusion protein but

instead have chromosome

gains, recurrent point

mutations and 11p15.5

$\quad$ allelic loss

$\because$

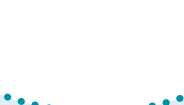

\section{OUTLOOK}

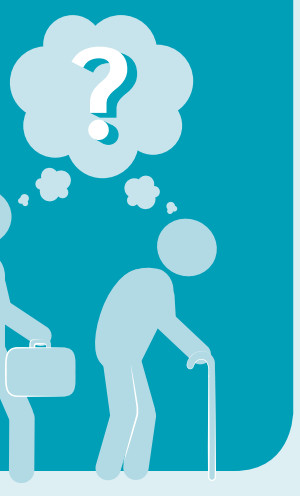

Chance of cure in children with high-risk, metastatic RMS is particularly poor, and all survivors of RMS can have long-term negative health effects, owing to intensive therapy. Several research avenues are seeking

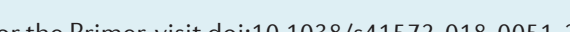

\section{MANAGEMENT}

Decades of study in large, cooperative-group clinical trials have improved the 5 -year overall survival of children with RMS to $>70 \%$. Currently, patients are stratified into high-, intermediate- and low-risk groups based on clinical, pathological and (increasingly) molecular parameters for tailored treatment. The primary tumour is removed by surgical resection and, in some patients, the tumour site is subjected to ionizing radiation. Disseminated (metastatic) disease is treated with multi-agent

chemotherapy, the intensity and duration of which

depends on the patient's risk group.

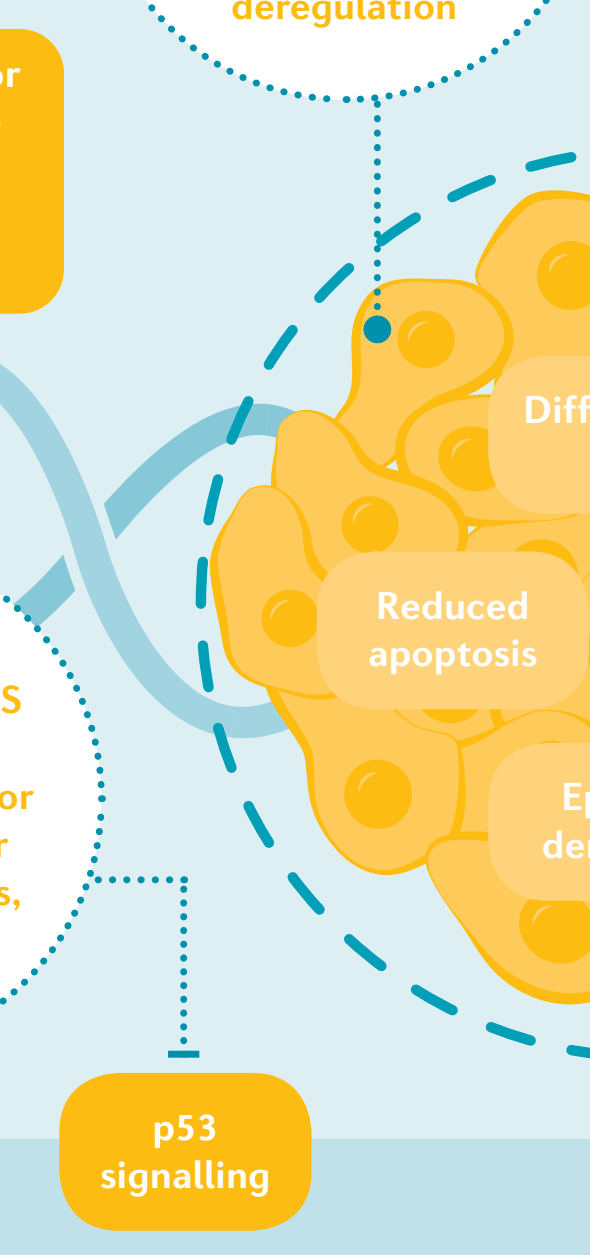

to address these problems. First, robust and objective biomarkers should be assessed, which could enable refined patient risk stratification. Second, functional and highthroughput laboratory approach may allow the identification of new therapies and targets. Third, prospective collection of patient-reported outcomes will enable long-term follow-up of the symptom experience and quality of life of people with RMS.
RMS can arise at almost any anatomical site, but FNRMS is more likely to arise in the head and/or neck and genitourinary sites. Signs and symptoms are associated with a soft tissue mass, with potential mass effect on adjacent organs. Although histopathological assessment is required for diagnosis, molecular features are increasingly being used in the diagnosis and classification of RMS. Approximately $20 \%$ of children have radiographic or clinical evidence of distant metastases at diagnosis. 